PROCEEDINGS OF THE

AMERICAN MATHEMATICAL SOCIETY

Volume 134, Number 8, Pages 2465-2472

S 0002-9939(06)08234-7

Article electronically published on February 3, 2006

\title{
SELF DELTA-EQUIVALENCE OF COBORDANT LINKS
}

\author{
YASUTAKA NAKANISHI, TETSUO SHIBUYA, AND AKIRA YASUHARA \\ (Communicated by Ronald A. Fintushel)
}

\begin{abstract}
Self $\Delta$-equivalence is an equivalence relation for links, which is stronger than the link-homotopy defined by J. Milnor. It is known that cobordant links are link-homotopic and that they are not necessarily self $\Delta$ equivalent. In this paper, we will give a sufficient condition for cobordant links to be self $\Delta$-equivalent.
\end{abstract}

\section{INTRODUCTION}

In this paper, all links will be assumed to be ordered and oriented, and they will be considered up to ambient isotopy.

A $\Delta$-move [6] is a local move on links as illustrated in Figure 1. If the three strands in Figure 1 belong to the same component of a link, we call it a self $\Delta$ move [11. Two links are said to be self $\Delta$-equivalent if one can be deformed into the other by a finite sequence of self $\Delta$-moves.

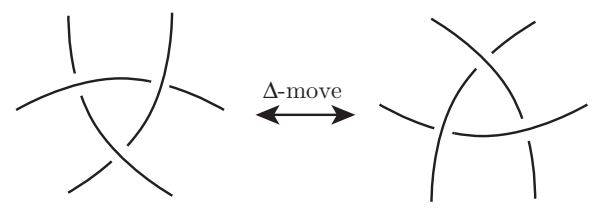

FiguRE 1.

Two links are said to be link-homotopic if one can be deformed into the other by a finite sequence of self-crossing changes [5]. Note that self $\Delta$-equivalence implies link-homotopy, i.e., if two links are self $\Delta$-equivalent, then they are link-homotopic.

Let $L_{i}=K_{i 1} \cup \cdots \cup K_{i n}(i=0,1)$ be $n$-component links. Two links $L_{0}$ and $L_{1}$ are cobordant if there is a disjoint union $\mathcal{A}=A_{1} \cup \cdots \cup A_{n}$ of $n$ annuli in $S^{3} \times[0,1]$ with $\left(\partial\left(S^{3} \times[0,1]\right), \partial A_{j}\right)=\left(S^{3} \times\{0\}, K_{0 j}\right) \cup\left(-S^{3} \times\{1\},-K_{1 j}\right)(j=1, \ldots, n)$, where $-X$ denotes $X$ with the opposite orientation. Then $\mathcal{A}$ is called a concordance between $L_{0}$ and $L_{1}$.

It is known that cobordism implies link-homotopy [1, 3. In [11, it is shown that every ribbon link is self $\Delta$-equivalent to a trivial link, and it is conjectured that if two links are cobordant, then they are self $\Delta$-equivalent. Nakanishi and Shibuya [9] give a counterexample for this conjecture. After that Nakanishi and Ohyama

Received by the editors October 19, 2004 and, in revised form, March 3, 2005.

2000 Mathematics Subject Classification. Primary 57M25.

Key words and phrases. $\Delta$-move, self $\Delta$-equivalence, link-homotopy, cobordant.

(C)2006 American Mathematical Society 
give a classification for 2-component links up to self $\Delta$-equivalence [7. By using their classification theorem, we have the following: (1) If 2-component links with linking numbers zero are cobordant, then they are self $\Delta$-equivalent. (2) The links illustrated in Figure 2 are cobordant and are not self $\Delta$-equivalent if $|p| \neq 0$. This implies that for any integer $p \neq 0$, there are two links with linking number $p$ such that they are cobordant and not self $\Delta$-equivalent. The case that $|p|=1$ is due to Nakanishi and Shibuya 9. The linking number is an obstruction for links which are cobordant to be self $\Delta$-equivalent. Our purpose is to find a sufficient condition for links which are cobordant to be self $\Delta$-equivalent.

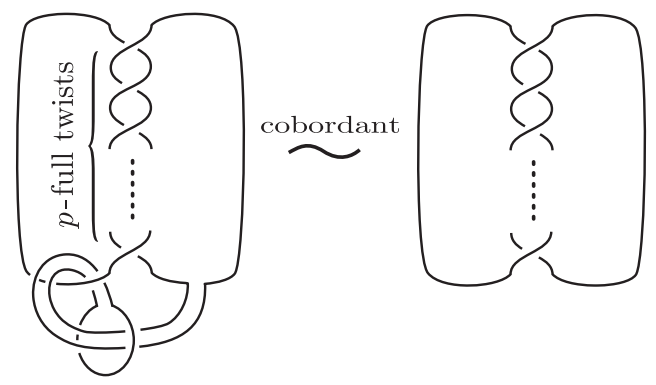

FiguRE 2.

Since any knot is (self) $\Delta$-equivalent to a trivial knot [6], any link is self $\Delta$ equivalent to a link each component of which is a trivial knot. For a 2-component link with trivial components, the following conditions are equivalent:

(1) The linking number is 0.

(2) It is link-homotopic to a trivial link.

(3) Each component is null-homotopic in the complement of the other component.

For links with $n$ trivial components $(n \geq 3)$, the conditions above are not equivalent. Note that $(3) \Rightarrow(2) \Rightarrow(1)$. So we have the following questions.

Questions. Let $L_{0}$ and $L_{1}$ be cobordant and their components all trivial.

(1) If the linking numbers of all 2-component sublinks of $L_{i}(i=0,1)$ are 0 , then are they self $\Delta$-equivalent?

(2) If $L_{0}$ and $L_{1}$ are link-homotopic to a trivial link, then are they self $\Delta$ equivalent?

(3) If each component $K_{i}$ of $L_{i}$ is null-homotopic in $S^{3} \backslash\left(L_{i}-K_{i}\right)(i=0,1)$, then are they self $\Delta$-equivalent?

In this paper, we give a negative answer to Question (1) and an affirmative answer to Question (3). Question (2) is still open (likely negative).

The following theorem gives us an affirmative answer to Question (3).

Theorem 1. Let $L_{0}$ and $L_{1}$ be links which are cobordant. If each component $K_{i}$ of $L_{i}$ is null-homotopic in $S^{3} \backslash\left(L_{i}-K_{i}\right)(i=0,1)$, then $L_{0}$ and $L_{1}$ are self $\Delta$ equivalent.

The following propositon gives a negative answer to Question (1).

Proposition 1. There exists a 3-component link with trivial components such that it is cobordant to Borromean rings and it is not self $\Delta$-equivalent to Borromean rings. 


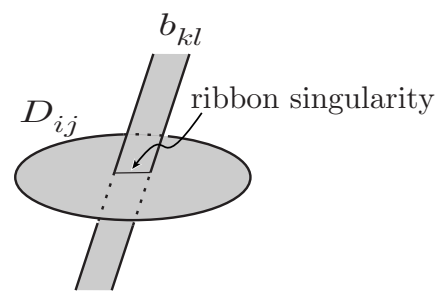

FIGURE 3.

\section{Proof of Theorem 1}

A concordance $\mathcal{A}$ in $S^{3} \times[0,1]$ between $L_{0} \subset S^{3} \times\{0\}$ and $L_{1} \subset S^{3} \times\{1\}$ is called a ribbon concordance [2] if the restriction to $\mathcal{A}$ of the projection $S^{3} \times[0,1] \rightarrow[0,1]$ is a Morse function with no minimal points. In this case we write $L_{0} \geq L_{1}$.

For a concordance $\mathcal{A}$ betwen $L_{0}$ and $L_{1}$, there is a concordance $\mathcal{A}^{\prime}$ between $L_{0}$ and $L_{1}$ such that $\mathcal{A}^{\prime}$ is ambient isotopic to $\mathcal{A}$ in $S^{3} \times[0,1]$ and $\mathcal{A}^{\prime} \cap\left(S^{3} \times[0,1 / 2]\right)$ (resp. $\left.\mathcal{A}^{\prime} \cap\left(S^{3} \times[1 / 2,1]\right)\right)$ is a ribbon concordance for $\mathcal{A}^{\prime} \cap\left(S^{3} \times\{1 / 2\}\right) \geq K_{0}$ (resp. $\left.\mathcal{A}^{\prime} \cap\left(S^{3} \times\{1 / 2\}\right) \geq K_{1}\right)$. Therefore, Theorem 1 follows directly the following theorem.

Theorem 2. Let $L_{0}$ and $L_{1}$ be links. If $L_{0} \geq L_{1}$ and each component $K$ of $L_{1}$ is null-homotopic in $S^{3} \backslash\left(L_{1}-K\right)$, then $L_{0}$ and $L_{1}$ are self $\Delta$-equivalent.

Proof. Suppose $L_{0}=K_{01} \cup K_{02} \cup \cdots \cup K_{0 n}$ and $L_{1}=K_{11} \cup K_{12} \cup \cdots \cup K_{1 n}$ are not self $\Delta$-equivalent. We choose $L_{0}$ up to self $\Delta$-equivalence so that the number of the maximal points for ribbon concordance $A_{1} \cup A_{2} \cup \cdots \cup A_{n}$ between $L_{0}$ and $L_{1}$ is minimum. It is known in 44 that $L_{0} \geq L_{1}$ if and only if $L_{0}$ is a band sum of $L_{1}$ and a trivial link. Hence, there exist a disjoint union $\bigcup_{i, j} D_{i j}$ of 2 disks $D_{11}, \ldots, D_{1 m_{1}}, \ldots, D_{n 1}, \ldots, D_{n m_{n}}$ and a disjoint union $\bigcup_{i, j} b_{i j}$ of 2-disks, bands, $b_{11}, \ldots, b_{1 m_{1}}, \ldots, b_{n 1}, \ldots, b_{n m_{n}}$ such that

(1) $L_{1} \cap\left(\bigcup_{i j} D_{i j}\right)=\emptyset$,

(2) $b_{k l} \cap\left(L_{1} \cup \bigcup_{i j} \partial D_{i j}\right)=b_{k l} \cap\left(K_{1 k} \cup \bigcup_{j} \partial D_{k j}\right)$ consists of disjoint two-arcs in $\partial b_{k l}$,

(3) $b_{k l} \cap\left(\bigcup_{i j} \operatorname{int} D_{i j}\right)$ consists of proper arcs in $b_{k l}$, which are called ribbon singularities (see Figure 3),

(4) $L_{0}=L_{1} \cup \bigcup_{i j}\left(\partial D_{i j} \cup \partial b_{i j}\right)-\operatorname{int}\left(\left(\bigcup_{i j} \partial b_{i j}\right) \cap\left(L_{1} \cup \bigcup_{i j} \partial D_{i j}\right)\right)$, and

(5) $K_{0 k}=K_{1 k} \cup \bigcup_{j}\left(\partial D_{k j} \cup \partial b_{k j}\right)-\operatorname{int}\left(\left(\bigcup_{j} \partial b_{k j}\right) \cap\left(K_{1 k} \cup \bigcup_{j} \partial D_{k j}\right)\right)$.

Note that the 2-disks $D_{i j}$ 's correspond to the maximal points for the ribbon concordance. Set $\mathcal{D}_{k}=\bigcup_{j} D_{k j}, \mathcal{D}=\bigcup_{k} \mathcal{D}_{k}, \mathcal{B}_{k}=\bigcup_{j} b_{k j}, \mathcal{B}=\bigcup_{k} \mathcal{B}_{k}$. We may suppose that $\mathcal{D}_{1} \neq \emptyset$. Since $K_{11}$ is null-homotopic in $S^{3} \backslash\left(L_{1}-K_{11}\right), K_{11}$ bounds a singular 2-disk $D_{0}$ in $L_{1}-K_{11}$ each singularity of which is a clasp [10] (see Figure 4). We may assume that $D_{0} \cap \mathcal{D}=\emptyset, \mathcal{B}$ is disjoint from the clasp singularities of $D_{0}$, and $\left(D_{0}-K_{11}\right) \cap \mathcal{B}$ consists of ribbon singularities.

Moreover, by sliding bands in $\mathcal{B}_{1}$ suitably [4, we may assume that $b_{11}$ connects $\partial D_{0}$ and $\partial D_{11}$, and that $b_{1 j}$ connects $\partial D_{1(j-1)}$ and $\partial D_{1 j}$, without changing the number of 2-disks in $\mathcal{D}$.

We deform $D_{0} \cup \mathcal{D}_{1} \cup \mathcal{B}_{1}$ into $D_{0}^{\prime} \cup \mathcal{D}_{1}^{\prime} \cup \mathcal{B}_{1}^{\prime}$ as illustrated in Figure 5 (a), (b) so that $D_{0}^{\prime} \cap \mathcal{B}_{1}^{\prime}=\emptyset$ and that each 2-disk in $\mathcal{D}_{1}^{\prime}$ contains at most a single ribbon singurality of $\mathcal{D}_{1}^{\prime} \cap \mathcal{B}_{1}^{\prime}$. Here we temporarily ignore $\mathcal{D}_{1}^{\prime} \cap \mathcal{B}_{k}(k \geq 2)$. Set $\mathcal{D}_{1}^{\prime}=$ 


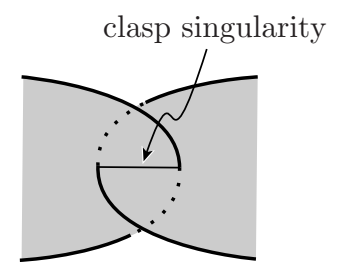

Figure 4.

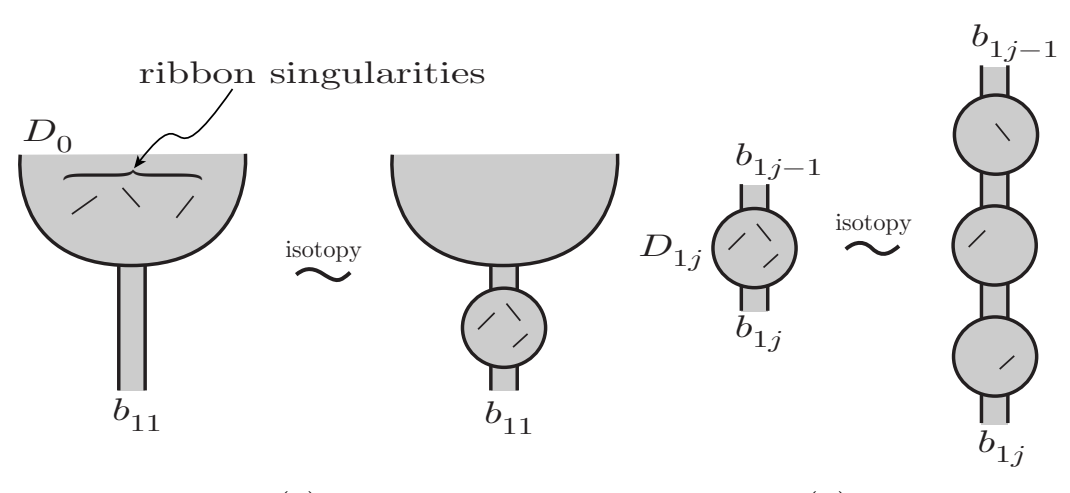

(a)

(b)

FIGURE 5 .

$D_{1} \cup D_{2} \cup \cdots \cup D_{m}, \mathcal{B}_{1}^{\prime}=b_{1} \cup b_{2} \cup \cdots \cup b_{m}$, and assume that $b_{1}$ connects $D_{0}^{\prime}$ and $D_{1}$, and that $b_{j}$ connects $D_{j-1}$ and $D_{j}(j \geq 2)$.

Claim. The deformations as illustrated in Figure 6 (a), (b) and (c) are realized by $\Delta$-moves.

Proof of Claim. Since the local move as illustrated in Figure 7 is realized by a single $\Delta$-move and ambient isotopies (for example, see [12]), the claim above follows Figure 8 (a), (b) and (c).

By combining the deformations as illustrated in Figure 6 (a), (b) and (c), we can change $D_{m} \cup b_{m}$ so that $D_{m} \cap \mathcal{B}_{1}^{\prime}=\emptyset$. Then we shrink $D_{m} \cup b_{m}$ into a part of $D_{m-1}$. In these deformations, the ambient isotopy class of $\left(L_{1}-K_{11}\right) \cup \bigcup_{k \geq 2}\left(\mathcal{D}_{k} \cup \mathcal{B}_{k}\right)$ is preserved, although $\mathcal{B}_{k}$ might be trailed by $D_{m}$. Thus we have a new band sum of $L_{1}$ and $\partial\left(\mathcal{D}_{1}^{\prime}-D_{m}\right) \cup \bigcup_{k \geq 2} \partial \mathcal{D}_{k}$ with bands $\left(\mathcal{B}_{1}^{\prime}-b_{m}\right) \cup \bigcup_{k \geq 2} \mathcal{B}_{k}^{\prime}$. Repeating these deformations, we have a band sum $L_{0}^{\prime}=K_{11} \cup K_{02}^{\prime} \cup \cdots \cup K_{0 n}^{\prime}$ of $L_{1}$ and $\bigcup_{k \geq 2} \partial \mathcal{D}_{k}$ with bands $\bigcup_{k \geq 2} \mathcal{B}_{k}^{\prime \prime}$ such that $\left(L_{1}-K_{11}\right) \cup \bigcup_{k \geq 2}\left(\mathcal{D}_{k} \cup \mathcal{B}_{k}^{\prime \prime}\right)$ is ambient isotopic to $\left(L_{1}-K_{11}\right) \cup \bigcup_{k \geq 2}\left(\mathcal{D}_{k} \cup \mathcal{B}_{k}\right)$. We note that $L_{0}^{\prime}$ is self $\Delta$-equivalent to $L_{0}$, and that $L_{0}^{\prime}$ and $L_{1}$ bound a ribbon concordance $\left(K_{11} \times I\right) \cup A_{2} \cup \cdots \cup A_{n}$. This contradicts the minimality of the number of maximal points for the ribbon concordance.

\section{Proof of Proposition 1}

Let $L_{0}$ be Borromean rings, and let $L_{1}$ be a link as illustrated in Figure 9 . Since $L_{0}$ and $L_{1}$ are cobordant, we will show that $L_{0}$ and $L_{1}$ are not self $\Delta$-equivalent. 
$=\frac{C+D=}{C}=$

(a)

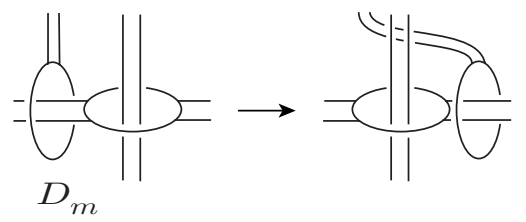

(b)

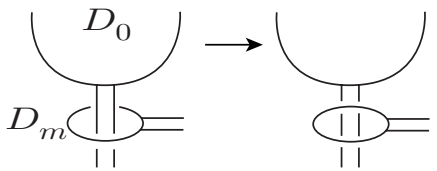

(c)

FiguRE 6.<smiles>CC1(C)CCC1(C)C1(C)C(C)(C)C(C)(C)C1(C)C</smiles>

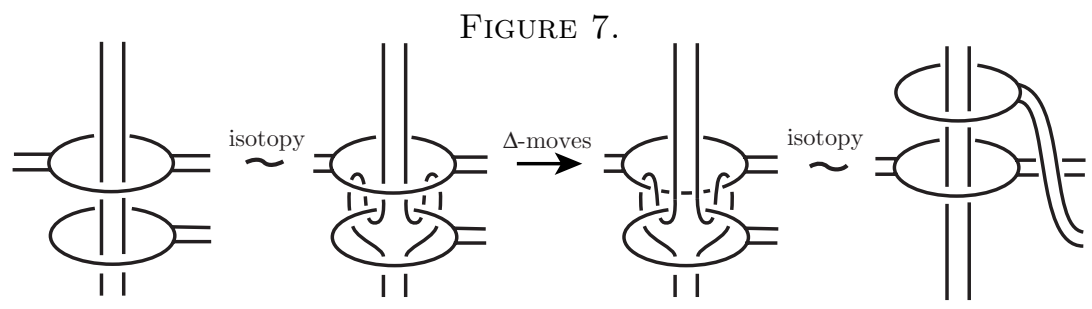

(a)

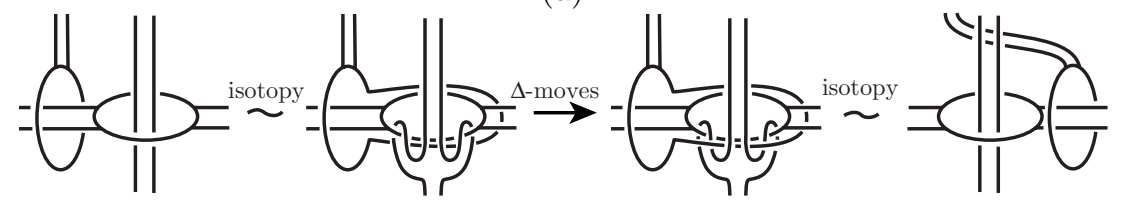

(b)
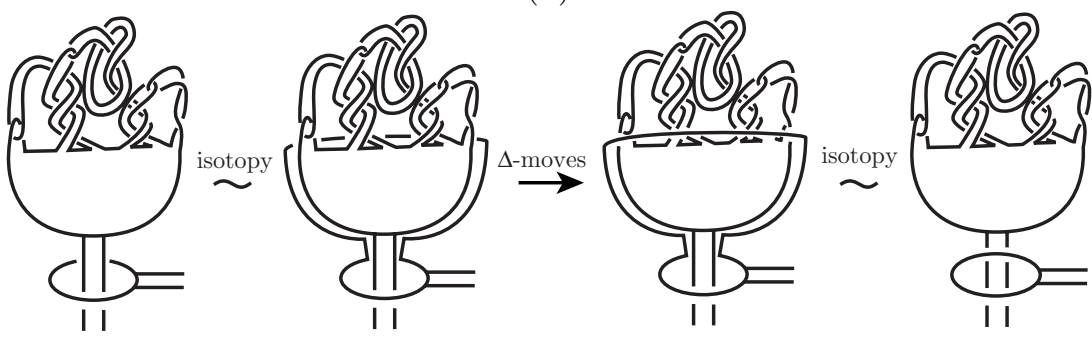

(c)

Figure 8. 


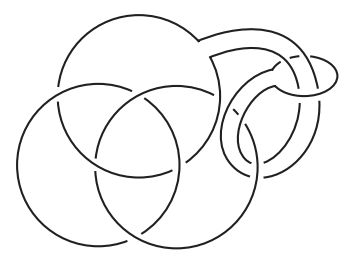

FiguRE 9.

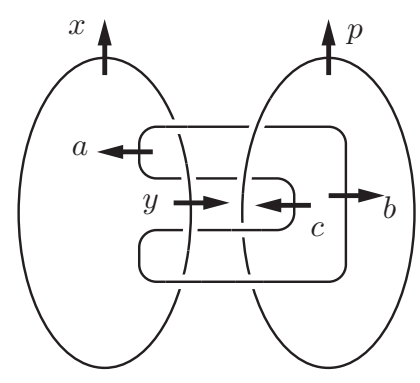

FiguRE 10

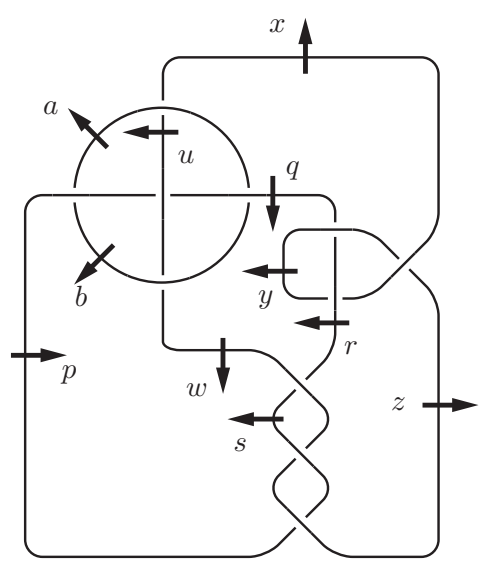

FIGURE 11

In order to prove this, we need the following proposition given in [8].

Proposition 2 ([8, Lemma 3.1]). If two $n$-component links are self $\Delta$-equivalent, then they have the same Alexander matrices modulo $\left(\left(1-t_{1}\right)^{2}, \ldots,\left(1-t_{n}\right)^{2}\right)$. Further, we have similar statements for the elementary ideals of deficiency greater than 0.

Proof of Proposition 1. We take a diagram of $L_{0}$ as illustrated in Figure 10. Then we have

$$
\pi_{1}\left(S^{3} \backslash L_{0}\right)=\left\langle a, b, c, p, x, y \mid \begin{array}{l}
y=a x a^{-1}, x=b^{-1} y b, p=b^{-1} c p c^{-1} b, \\
b=p a p^{-1}, c=y^{-1} b y, a=y p^{-1} c p y^{-1}
\end{array}\right\rangle .
$$

This can be shown to be isomorphic to $\left\langle a, p, x \mid S_{1}, S_{2}\right\rangle$, where

$$
\begin{aligned}
& S_{1}=p a^{-1} p^{-1} a x a^{-1} p a p^{-1} x^{-1} \\
& S_{2}=a^{-1} p^{-1} a x^{-1} a^{-1} p^{-1} a x a^{-1} p^{-1} x^{-1} a^{-1} p a^{-1} p^{-1} a x a^{-1} p a p^{-1} .
\end{aligned}
$$

Hence the Alexander matrix is equivalent to the following matrix modulo $\left((a-1)^{2},(p-1)^{2},(x-1)^{2}\right)$ :

$$
\left(\begin{array}{ccc}
(p-1)(x-1) & -(a-1)(x-1) & 0 \\
(p-1)(x-1) & -a^{-1}(a-1)(p-1)(x-1) & (a-1)(p-1)
\end{array}\right) .
$$

So we have

$$
E_{1} \equiv(0) \bmod \left((a-1)^{2},(p-1)^{2},(x-1)^{2}\right)
$$


On the other hand, we take a diagram of $L_{1}$ as illustrated in Figure 11. Then we have

$$
\pi_{1}\left(S^{3} \backslash L_{1}\right)=\left\{\begin{array}{l|l}
x, y, z, w, u, & \begin{array}{l}
y=r^{-1} a r, z=x^{-1} y x, w=s z s^{-1}, u=b w b^{-1} \\
x=a^{-1} u a, b=q^{-1} a q, a=p b p^{-1}, q=u p u^{-1} \\
r=y, p, q, r, s
\end{array} \\
r=y q y^{-1}, s=w^{-1} r w, p=z^{-1} s z
\end{array}\right\} .
$$

This can be shown to be isomorphic to $\left\langle y, w, a, q \mid R_{1}, R_{2}, R_{3}\right\rangle$, where

$$
\begin{aligned}
& R_{1}=q^{-1} y^{-1} a y q y^{-1} \\
& R_{2}=y q y^{-1} w a^{-1} q^{-1} a q w^{-1} q^{-1} a^{-1} q a y a^{-1} q^{-1} a q w q^{-1} a^{-1} q a w^{-1} y q^{-1} y^{-1} w^{-1} \\
& R_{3}=a q w q^{-1} a^{-1} q a^{-1} q^{-1} a q w^{-1} q^{-1} a^{-1} q a y^{-1} a^{-1} q^{-1} a q w q^{-1} a^{-1} q a w^{-1} y q .
\end{aligned}
$$

The Alexander matrix is modulo $\left((a-1)^{2},(q-1)^{2},(y-1)^{2}\right)$ equivalent to

$$
\left(\begin{array}{cccc}
-a(q-1)-1 & 0 & 1 & y(a-1) \\
y(q-1)+1 & -1 & 0 & -y(y-1) \\
0 & -y a q(q-1)(a-1) & y a(q-1)(y-1) & (y-1)(a-1)(q-1)
\end{array}\right)
$$

By fundamental deformations of presentation matrices up to modulo $\left((a-1)^{2}\right.$, $\left.(q-1)^{2},(y-1)^{2}\right)$, we have

$$
((q-1)(y-a) \quad(y-1)(a-1)(q-1)) .
$$

Hence we have

$$
E_{1} \equiv((q-1)(y-a)) \bmod \left((a-1)^{2},(q-1)^{2},(y-1)^{2}\right) .
$$

By Proposition 2, we have the conclusion.

\section{REFERENCES}

1. C. H. Giffen, Link concordance implies link homotopy, Math. Scand. 45(1979), 243-254. MR $0580602(82 \mathrm{a}: 57005)$

2. C. McA. Gordon, Ribbon concordance of knots in the 3-sphere, Math. Ann. 257(1981), 157170. MR0634459 (83a:57007)

3. D. L. Goldsmith, Concordance implies homotopy for classical links in $M^{3}$, Comment. Math. Helv. 54(1979),347-355. MR0543335 (80h:57006)

4. A. Kawauchi, T. Shibuya and S. Suzuki, Descriptions on surfaces in four-space, I. Normal forms, Math. Sem. Notes, Kobe Univ., 10 (1982), 75-125. MR0672939 (84d:57017)

5. J. Milnor, Link groups, Ann. Math., 59 (1954) 177-195. MR0071020 (17:70e)

6. H. Murakami and Y. Nakanishi, On a certain move generating link-homology, Math. Ann., 284(1989), 75-89. MR0995383 (90f:57007)

7. Y. Nakanishi and Y. Ohyama, Delta link homotopy for two component links. III, J. Math. Soc. Japan 55(2003), 641-654. MR1978214 (2004a:57009)

8. Y. Nakanishi and T. Shibuya, Relations among self delta-equivalence and self sharpequivalences for links, Knots in Hellas '98 (Delphi), 353-360, Ser. Knots Everything, 24, World Sci. Publishing, River Edge, NJ, 2000. MR1865717 (2002h:57014)

9. Y. Nakanishi and T. Shibuya, Link homotopy and quasi self delta-equivalence for links, J. Knot Theory Ramifications 9(2000), 683-691. MR.1762762 (2001d:57011)

10. T. Shibuya, Some relations among various numerical invariants for links, Osaka J. Math. 11(1974), 313-322. MR0353295(50:5779) 
11. T. Shibuya, Self $\Delta$-equivalence of ribbon links, Osaka J. Math, 33(1996), 751-760. MR,1424684 (97k:57012)

12. K. Taniyama and A. Yasuhara, Clasp-pass moves on knots, links and spatial graphs, Topology Appl. 122(2002), 501-529. MR1911697 (2003g:57012)

Department of Mathematics, Kobe University, Nada, Kobe 657-8501, Japan

E-mail address: nakanisi@math.kobe-u.ac.jp

Department of Mathematics, Osaka Institute of Technology, Omiya 5-16-1, Asahi, OSAKA 535-8585, JAPAN

E-mail address: shibuya@ge.oit.ac.jp

Department of Mathematics, Tokyo Gakugei University, Nukuikita 4-1-1, Koganei, TOKYO 184-8501, JAPAN

E-mail address: yasuhara@u-gakugei.ac.jp 